\title{
A link between nonlinear self-organization and dissipation in drift-wave turbulence
}

\author{
P. Manz $^{1}$, G. Birkenmeier ${ }^{2}$, M. Ramisch ${ }^{3}$, U. Stroth ${ }^{2}$ \\ 1 Center for Momentum Transport and Flow Organization, \\ University of California at San Diego, San Diego, California 92093, USA \\ 2 Max-Planck-Institut für Plasmaphysik, Association Euratom-IPP, 85748 Garching, Germany \\ 3 Institut für Plasmaforschung, Universität Stuttgart, 70569 Stuttgart, Germany
}

(Dated: January 30, 2013)

\begin{abstract}
Structure formation and self-organization in two-dimensional drift-wave turbulence show up in many different faces. Fluctuation data from a magnetized plasma are analyzed and three mechanisms transferring kinetic energy to large-scale structures are identified. Beside the common vortex merger, clustering of vortices constituting a large-scale strain field and vortex thinning, where due to the interactions of vortices of different scales larger vortices are amplified by the smaller ones, are observed. The vortex thinning mechanism appears to be the most efficient one to generate large scale structures in drift-wave turbulence. Vortex merging as well as vortex clustering are accompanied by strong energy transfer to small-scale noncoherent fluctuations (dissipation) balancing the negative entropy generation due to the self-organization process.
\end{abstract}

PACS numbers:

\section{INTRODUCTION}

Due to the strong background magnetic field, the dynamic of turbulence in fusion experiments is nearly bounded to the plane perpendicular to the magnetic field and therefore nearly two-dimensional. Two dimensional turbulence is not just a dimensional reduced version of three-dimensional turbulence because new conservation laws arise giving two-dimensional turbulence its own phenomenology. A review of recent developments in twodimensional turbulence can be found in Ref. [1]. Where kinetic energy cannot be dissipated by viscosity in two dimensions it is transferred to larger scales in motion by the inverse energy cascade [1]. The cascade is defined here as a process whereby nonlinear interactions conservatively transport energy in wavenumber space $k$. In recognition of the historical importance of the turbulent cascade, nonlocal transfer in $k$-space which in general is not consistent with the concept of a cascade is called nevertheless (nonlocal) cascade, here. Commonly the physical mechanism behind the inverse energy cascade is understood as a sequence of merging eddies of similar size producing larger and larger vortices. It depicts a local inverse transfer for kinetic energy in $k$-space. Paret and Tabeling found that this mechanism is not the only possibility for an inverse energy transfer. Alternatively, the cascade could be rather driven by a clustering mechanism involving same sign vortices [2]. Thus, as in three-dimensional turbulence, 2D-turbulence is self-organized by aligning vortices in a large-scale strain field and energy is transferred to larger scales by a clustering process. Another mechanism called vortex thinning has been sugessted by Chen et al. [3]. Here the interaction of structures of different scales is responsible for the inverse energy transfer and the energy is transferred between non-contiguous spectral ranges (which means also nonlocal in $k$-space) [3].

In general turbulent flows contain both an organized part (the coherent vortices) and a random part, where the fluctuations are not organized by coherent vortices [4]. Disturbances are growing leading to the formation of vortical structures. Therefore, energy is transferred from the mean field (background gradients, flows etc.) to coherent energy which represents the energy in the vortical structures. Due to vortex interactions the energy is redistributed among different scales of motion, which let the coherent part of the turbulence to be expected to be responsible for the nonlinear energy transfer. Than the entire turbulence would take part within the coherent part of the turbulence and there would be no physical need for random fluctuations to appear.

The self-organization of turbulence leads to a reduction of the degrees of freedom and therefore to a negative entropy generation whereas energy is transferred to larger and larger scales. In three-dimensional turbulence this is balanced by the direct cascade, where the large coherent structures are getting smaller and smaller due to vortex interaction and pass their energy finally to the random field. By this mechanism the energy is dissipated. The information necesarry for time reversal is not lost, instead it is transferred to the smallest scales in the system where it is invisible for practical purpose. In two-dimensional turbulence the energy is transferred to larger scales and a dissipation process like in three dimensions is not available. But also for two-dimensional turbulence the energy must be dissipated and the energy has to be transferred finally to the small-scale random field. It appears reasonable to suggest that there is an interaction between the coherent and the random part of two-dimensional turbulence.

In previous investigations [5] in a toroidally confined low-temperature plasma in the stellerator experiment TJ-K [6] the energy transfer between turbulent vortices of different scales has been investigated experimentally by means of a two-field model, which is tailored to driftwave turbulence [7]. Potential fluctuations, from which 
the turbulent $E \times B$ flow can be derived, have been measured with a two-dimensional probe array. It has been found that the energy and the enstrophy are transferred in oposite directions in $k$-space as expected for two-dimensional fluid turbulence $[5,8]$. The energy cascades to larger scales with the capability of driving largescale flows. Nonlocal transfer has been shown to play an important role in the inverse cascade and the results supported the vortex thinning picture as proposed by Chen et al. [3]. However, it has not been distinguished if the energy has been contained in coherent structures as eddies or noncoherent structures as random fluctuations or large-scale strain fields. In this work the generation mechanisms of large-scale turbulent structures as well as the dissipation mechanisms in a close to two-dimensional system will be investigated in detail considering the energy transfer between different scales of coherent and noncoherent motion providing the possibility to distinguish the different mechanisms of large-scale turbulent structure formation.

\section{EXPERIMENTAL SETUP}

The experimental conditions are in line with the assumptions made for the Hasegawa-Wakatani model. The ion temperature of TJ-K plasmas is less than $1 \mathrm{eV} \mathrm{[9]} \mathrm{and}$ the electron temperature is constant at about $T_{e}=8 \mathrm{eV}$. The working gas was helium at a neutral gas pressure of $p=4 \cdot 10^{-5}$ mbar. Plasma is generated by microwaves

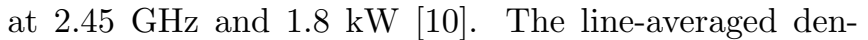
sity was about $\bar{n}=10^{17} \mathrm{~m}^{-3}$ and the magnetic field strength was $B=72 \mathrm{mT}$. The excellent accessibility for probe arrays provides the unique possibility to measure wavenumber spectra in $2 \mathrm{D}$ over long time series. Potential fluctuations were measured with an $8 \times 8$ array of Langmuir probes with a spatial resolution of $1 \mathrm{~cm}$. Details on the diagnostic can be found in Ref. [11]. The floating potential fluctuations $\tilde{\phi}$ are interpreted as plasma potential fluctuations, which has been shown to be valid for TJ-K plasmas [12]. All lengths are normalised to the drift-scale parameter $\rho_{s}=\sqrt{\left(m_{i} T_{e}\right)} /(e B)$ and times to $\rho_{s} / c_{s}$. Here $c_{s}=\sqrt{T_{e} / m_{i}}$ is the sound speed, $m_{i}$ the ion mass. The normalised fluctuations are $\tilde{\phi} \rightarrow\left(L_{n} / \rho_{s}\right)\left(e \tilde{\phi} / T_{e}\right)$. A radially movable probe measured the equilibrium density $n_{e}$ and temperature profile $T_{e} \cdot \rho_{s}$ is set from the equilibrium measurement to 1.2 $\mathrm{cm}$ and the density scale length $L_{n}=n_{e} / \nabla n_{e}$ is about $0.08 \mathrm{~m}$ and constant across the density gradient region. From the deduced collisionality $\nu$ and a typical parallel wavenumber of $k_{\|} \approx 0.41 \mathrm{~m}^{-1}$ [13], the adiabatic parameter is estimated to $C=k_{\|}^{2} / \nu \approx 1$. A similar discharge as in Ref. [8] is analysed. More experimental details can be found there.

\section{COHERENT AND NONCOHERENT STRUCTURES}

The Reynolds decomposition, where the measure is decoposed in a mean and a fluctuating part, has been the most applied statistical method to investigate turbulent flows. To study coherent structures the Reynolds decomposition can be extended to the triple decomposition [4]

$$
\varphi=\langle\varphi\rangle+\varphi_{c}+\varphi_{n}
$$

where any quantity as the stream function $\varphi$ here is decomposed in its mean, the coherent and noncoherent (random) contribution. There exist several definitions of coherent structures in literature. Here we use an identification going back to Okubo and Weiss [14, 15]. The Weiss number is $\operatorname{tr}\left((\nabla \otimes \mathbf{u})^{2}\right)-(\operatorname{tr}(\nabla \otimes \mathbf{u}))^{2}$ which is given by

$$
\mathcal{Q}=\frac{1}{2}\left(\sigma^{2}-\omega^{2}\right)
$$

where

$$
\sigma^{2}=\left(\partial_{x} \tilde{v}_{x}-\partial_{y} \tilde{v}_{y}\right)^{2}+\left(\partial_{y} \tilde{v}_{x}+\partial_{x} \tilde{v}_{y}\right)^{2}
$$

is the rate of deformation (this is the symmetric part of the stress tensor $\nabla \otimes \mathbf{u}$ ). The first term in Eq. (3) is called stretching strain, it includes both elongation and compression. The second term in Eq. (3) results due shear effects and is called shear deformation, here. $\omega=\nabla^{2} \varphi$ is the rotation called vorticity (this is the asymmetric part of the stress tensor $\nabla \otimes \mathbf{u}$ ), and $\tilde{v}_{x}=\partial_{y} \varphi, \tilde{v}_{y}=-\partial_{x} \varphi$ are the velocity components in the radial $x$ and poloidal $y$ directions in slab geometry, respectively. $\varphi$ is the stream function, which is directly proportional to the plasma potential $\phi$ assuming the $E \times B$ velocity as the dominant velocity and the magnetic field $B$ constant. When $\mathcal{Q}$ is positive the motion is hyperbolic in character and when negative, the motion is elliptical [15]. Hyperbolic motion corresponds to strain or deformation dominated regions, where elliptical regions are vorticity or rotation dominated. Therefore as it has been proven by Weiss [15], $\mathcal{Q}$ measures whether two particles will separate $(\mathcal{Q}>0)$ or not $(\mathcal{Q}<0)$ when following the frozen streamlines. In this way, a flow can be separated into structures and fluctuation, and we identify non-separating trajectories $(\mathcal{Q}<0)$ as belonging to coherent structures [16].

If we use the stream function the Weiss number $\mathcal{Q}$ reduces to

$$
\mathcal{Q}=\left(\partial_{x} \partial_{y} \varphi\right)^{2}-\left(\partial_{x}^{2} \varphi\right)\left(\partial_{y}^{2} \varphi\right)
$$

The first term is always positive and it is exactly one half of the stretching strain (first term in Eq. (3)). The stretching or compression always tries to decorrelate the turbulence in the sense that it increases the Weiss number $\mathcal{Q}$ which might result in a transition from a coherent structure $(\mathcal{Q}<0)$ to an open-streamline vorticity concentration $(\mathcal{Q}>0)$. This is called straining-out. It 
appears as the second term of Eq. (4) and means that the circular motion is stabilizing the turbulent structure. But the second term does not only result from the vorticity, it comes from the shear deformation (second term in Eq. (3)) and the vorticity in the same amount. For a negative Weiss number $\mathcal{Q}<0$ the vorticity must compensate the stretching deformation by the term $\left(\partial_{x}^{2} \varphi\right)\left(\partial_{y}^{2} \varphi\right)$, which also results in a stabilizing contribution in the same amount from the shearing deformations. This means, as long as the turbulent structure remains as a structure $(\mathcal{Q}<0)$, the shear is stabilizing.

In literature turbulent structures appear in a different terminology, they are for example called vortices, eddies, blobs or filaments and these terms are used interchangeable. However, there are sophisticated differences between them. We used the following definitions: vortices are concentrations of vorticity. Therefore all structures observed here are vortices. Eddies exhibit closed streamlines. Therefore coherent structures with $\mathcal{Q}<0$ are defined as eddies here. Filaments and sheet-like vortices exhibit non-closed streamlines with $\mathcal{Q}>0$, which are both noncoherent. The difference between filaments and sheet-like structures is given by their extent in direction parallel to the magnetic field. Filaments are finite structures, sheet-like vortices are infinite. From the measurements presented here they cannot be distinguished. As in general drift-waves exhibit finite parallel wavenumber they correspond to filaments but only if the fluctuations are noncoherent. Noncoherent structures with $\mathcal{Q}>0$ are defined as filaments, here. This definition is in line with the definitions in Refs. [17-19]. It should not be confused with the definition the blob community [20] use.

\section{SPECTRAL ENERGY TRANSFER}

A simple model to describe $E \times B$ flow and density perturbations for collisional drift waves is constituted by the Hasegawa-Wakatani equations [21, 22]. The direction of the energy and enstrophy transfer of the HasegawaWakatani turbulence has been shown analytically by Gang et al. [23] to be in the same direction as in twodimensional fluid turbulence. The diagnostic to calculate the energy transfer from the measurements is that of Camargo et al. [7]. It is an expansion for drift-wave turbulence of an energy transfer diagnostic originally developed for fluid turbulence by Domaradzki [24].

In a Fourier decomposition in terms of wavenumbers $k$, waves (or modes) can interact that satisfy the constraint $k=k_{1}+k_{2}$. The time evolution of the kinetic energy $E^{V q}$ of the coherent $(q=c)$ or noncoherent $(q=n)$ potential fluctuation field can be separated in linear and nonlinear terms, whereas the nonlinear terms have our main attention:

$$
\frac{\partial E^{V q}(\mathbf{k})}{\partial t}=\text { linear terms }+\sum_{\mathbf{k}_{\mathbf{1}}} T^{V q r s}\left(\mathbf{k} \leftarrow \mathbf{k}_{\mathbf{1}}\right)
$$

Here $\sum_{\mathbf{k}_{1}} T^{V q r s}\left(\mathbf{k} \leftarrow \mathbf{k}_{\mathbf{1}}\right)$ is the nonlinear transfer function which describes the nonlinear energy transfer to or from the mode $\mathbf{k}$ by the interaction with the modes $\mathbf{k}_{1}$ and $\mathbf{k}_{2}=\mathbf{k}-\mathbf{k}_{1}$. The spectral transfer of the fluid kinetic energy from mode $\mathbf{k}_{1}$ to mode $\mathbf{k}$ is given by $[7,25]$

$T^{V q r s}\left(\mathbf{k} \leftarrow \mathbf{k}_{\mathbf{1}}\right)=-2\left(k_{x} k_{1 y}-k_{1 x} k_{y}\right) k_{2}^{2} \operatorname{Re}\left\langle\phi_{\mathbf{k q}_{\mathbf{q}}}^{*} \phi_{\mathbf{k}_{\mathbf{1}} \mathbf{r}} \phi_{\mathbf{k}_{\mathbf{2}} \mathbf{s}}\right\rangle$,

where $q, r, s \in\{c, n\}$ denote the coherent and noncoherent potential field, respectively. The bispectrum $\left\langle\phi_{\mathbf{k q}}^{*} \phi_{\mathbf{k}_{1} \mathbf{r}} \phi_{\mathbf{k}_{\mathbf{2}} \mathbf{s}}\right\rangle$ only exhibits a finite value if all three waves $k, k_{1}$ and $k_{2}$ are phase-locked over some time. Therefore these waves have to be coherently coupled. The distinction between coherent and noncoherent fluctuating fields distinguishes between elliptical (closed) and hyperbolical (open) streamline configuarations. It does not give any information about the coherency of the coupling process between these modes. There is a difference between coherent structures and coherent nonlinear coupling. The asterisk denotes the complex conjugate and Re is the real part of a complex number. The factor $k_{x} k_{1 y}-k_{1 x} k_{y}=\mathbf{b}\left(\mathbf{k} \times \mathbf{k}_{\mathbf{1}}\right)$ is intrinsically two dimensional and follows from the nonlinearity. Here $\mathbf{b}$ is the unit vector in direction of the magnetic field. Thus a two dimensional treatment of the estimation of the energy transfer is essentially necessary and a $1 \mathrm{D}$ adaptation be taken with great caution. In the following analysis the ensemble average $\langle\cdot\rangle$ of these equations is taken. The method has been tested successfully on Hasegawa- Wakatani simulations [5] and it was used for the investigations of the dual cascade [5] and turbulence zonal flow interactions [26] on TJ-K. In difference to the previous investigations $[5,26]$ the method is applied to the potential field, decomposed in coherent and noncoherent structures to investigate the nonlinear interaction of both fields.

\section{TOTAL ENERGY TRANSFER}

The data analysis procedure is as follows. First the Weiss number $\mathcal{Q}(\phi(x, y))$ is calculated using Eq. (3) at every point in time. Then the potential field is decomposed in coherent and noncoherent structures, where

$$
\begin{gathered}
\phi_{c}(x, y)=\left\{\begin{array}{cc}
\phi(x, y) & \mathcal{Q}<0 \\
0, & \mathcal{Q} \geq 0
\end{array}\right. \\
\phi_{n}(x, y)=\left\{\begin{array}{cc}
0 & \mathcal{Q}<0 \\
\phi(x, y), & \mathcal{Q} \geq 0
\end{array}\right.
\end{gathered}
$$

Afterward both potential fields are Fourier transformed into the two-dimensional wave number space and the energy transfer functions are estimated according to Eq. (6). The results are summarized in Fig. 1. Largescale coherent structures $(k<2.5)$ are driven by coherent structures $\left(\sum_{\mathbf{k}_{1}} T^{V c c n}>0\right.$ and $\left.\sum_{\mathbf{k}_{1}} T^{V c c c}>0\right)$. Also $\sum_{\mathbf{k}_{\mathbf{1}}} T^{V c c n}$ and $\sum_{\mathbf{k}_{\mathbf{1}}} T^{V c n c}$ result from the same 


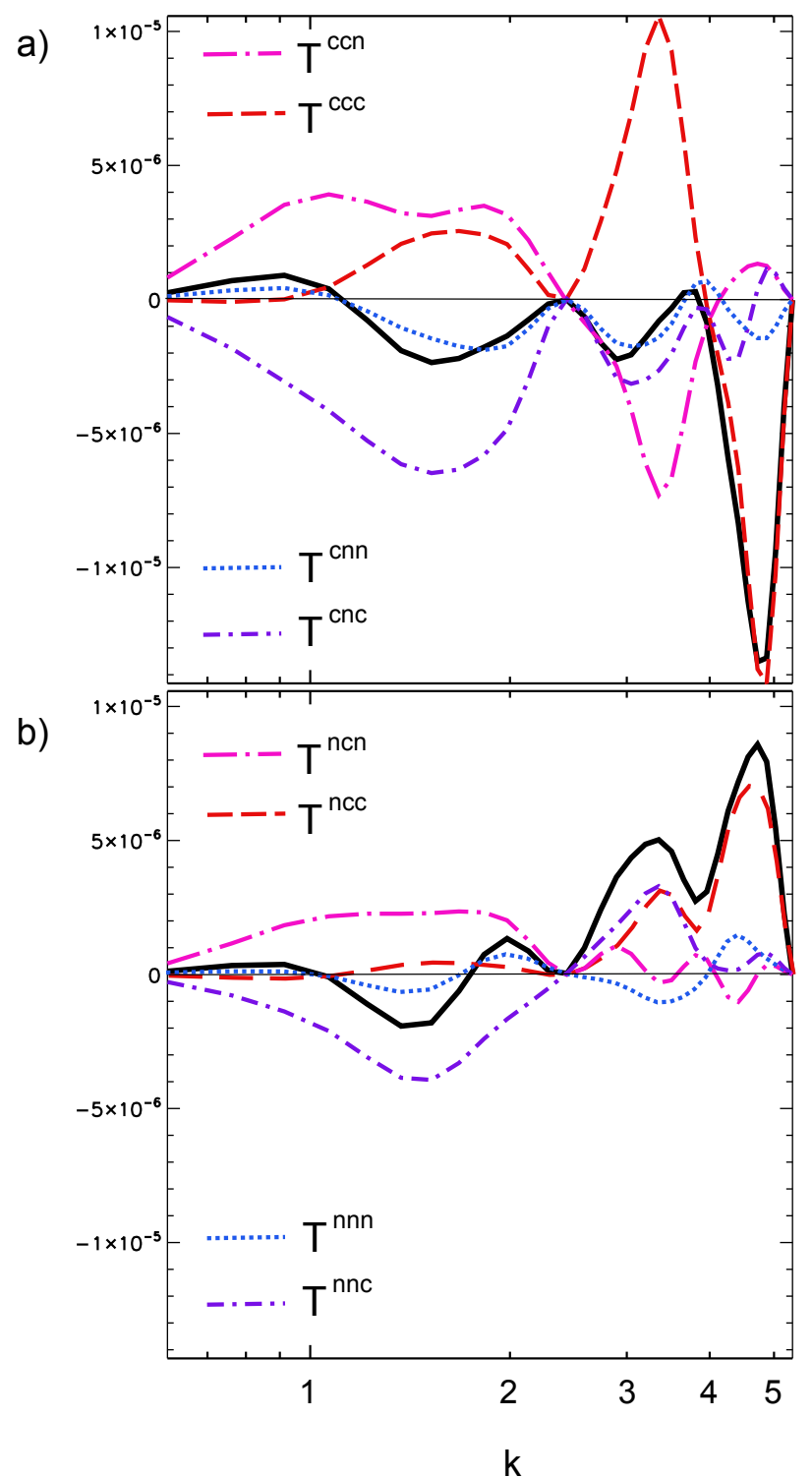

FIG. 1: Energy transfer into coherent $T^{V \text { crs }}$ (a) and noncoherent $T^{V n r s}$ (b) potential fluctuations. The solid black lines depict the total energy transfer $\sum_{r, s} T^{V c r s}$ and $\sum_{r, s} T^{V n r s}$.

coupling (two coherent and one noncoherent structures), they distinguish the source of the energy transfer into the coherent field. Both show the energy transfer into the coherent field, but $\sum_{\mathbf{k}_{1}} T^{V c c n}$ gives the energy coming from the coherent field and $\sum_{\mathbf{k}_{1}} T^{V c n c}$ gives the energy from the noncoherent (random) field. The energy in the large scale coherent fluctuations $\left(\sum_{\mathbf{k}_{1}} T^{V c n c}<0\right.$ for $k<2.5)$ is transferred directly to the random fluctuations $\left(\sum_{\mathbf{k}_{1}} T^{V n c c}>0\right.$ for $\left.k>2.5\right)$. It appears as the main drive of random fluctuations at small scales and consequently represents dissipation. A large-scale strain field is generated $\left(\sum_{\mathbf{k}_{1}} T^{V n c n}>0\right.$ with $\left.k<2.5\right)$ which is in turn balanced by a direct energy cascade of noncoherent fluctuations $\sum_{\mathbf{k}_{\mathbf{1}}} T^{V n n c}$ as in three-dimensions.

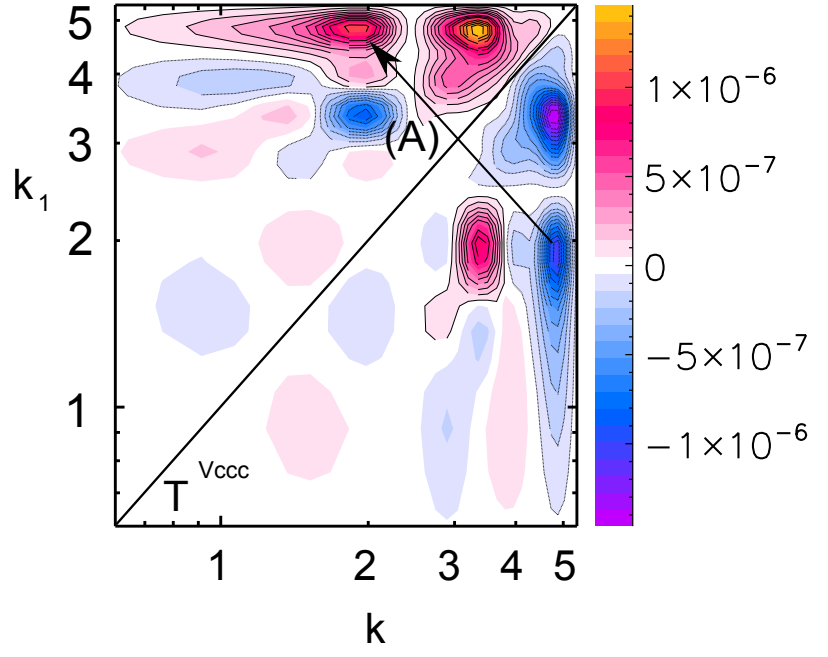

FIG. 2: Kinetic energy transfer between coherent structures. a)

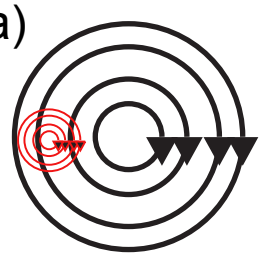

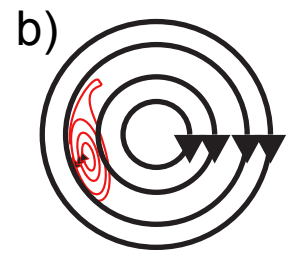

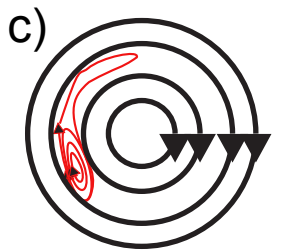

FIG. 3: Schmatics of the vortex thinning process. A smaller eddy in the shear of a larger one (a) is elongated and tilted (b). Finally the smaller eddy is subject to a straining-out process.

In the next three sections the energy transfer will be investigated and discussed in more detail. The results will be shown as in Refs. [5, 7]. $T\left(\mathbf{k} \leftarrow \mathbf{k}_{\mathbf{1}}\right)$ forms a four-dimensional quantity depending on $\left(k_{x}, k_{y}, k_{1 x}, k_{1 y}\right)$, with the constraint $\mathbf{k}=\mathbf{k}_{\mathbf{1}}+\mathbf{k}_{\mathbf{2}}$. Hence, for a graphical representation, sums of all contributions at given $|k|$ and $\left|k_{1}\right|$ have been taken and divided by the number of contributions.

\section{VORTEX THINNING}

Figure 2 shows the kinetic energy transfer into or from the coherent field in the $k-k_{1}$ plane. As the interactions are among coherent structures it depicts the energy transfer between different scales of eddies. Nonlinear interactions in general conservatively transport energy in wavenumber space $k$ and $T^{V q r s}\left(\mathbf{k} \leftarrow \mathbf{k}_{\mathbf{1}}\right)=$ $-T^{\text {Vrqs }}\left(\mathbf{k}_{\mathbf{1}} \leftarrow \mathbf{k}\right)$. Insofar no cross-field transfer is involved and the energy transfer is restricted to interaction within a subfield as $T^{V c c c}$ or $T^{V n n n}$ the energy should be conserved. The energy conservation is reflected in the antisymmetric behavior around the line given by $k=k_{1}$, the energy transfer is as expected conservative. 


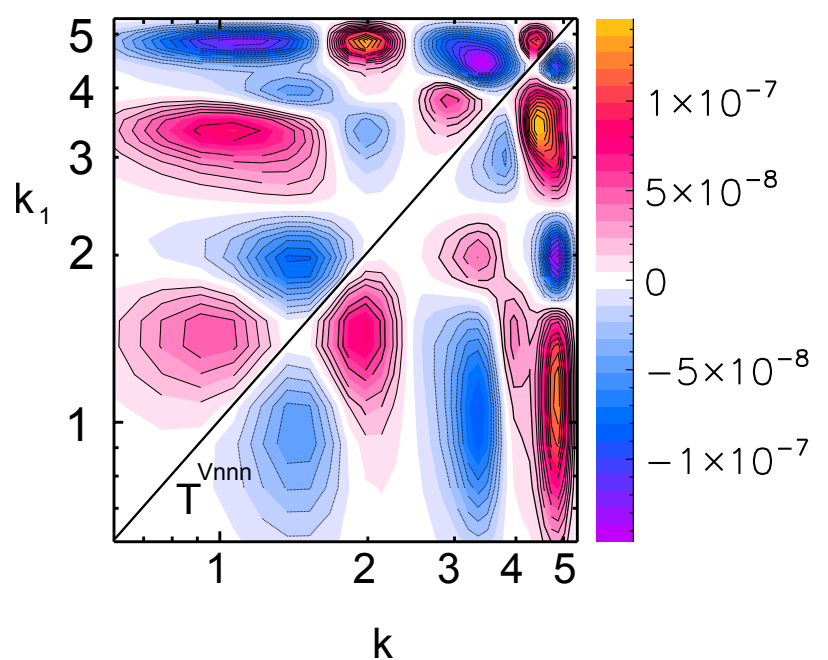

FIG. 4: Kinetic energy transfer between noncoherent structures.

Compared to the energy transfer including noncoherent fluctuations (Figs. 4, 5 and 8) the energy transfer between eddies is strongest in amplitude and therefore eddies dominate the total energy transfer. As seen in a previous investigation [5] the energy is dominated by energy transfer from the smallest scales $(k>4)$ into larger scales $(k<4)$ by nonlocal interactions (arrow (A) in Fig.2).

Figure 3 shows an artist view of this process. Due to the shear induced by the larger eddy the smaller one will be tilted and elongated [27] (3b). Since the circulation of an eddy is conserved, the velocity around the eddy is lowered and its energy is reduced [3, 28]. At the same time, the velocity of the eddy is now mainly directed such, that it reinforces the large-scale strain $[3,28]$. Thus energy is transferred from the smaller structure into the larger one by an elongation and tilting (and thinning) of the smaller structure. This process is effective, if the scales of the interacting structures are clearly different resulting in the pronounced nonlocality of this process in $k$-space. In the case of large-scale shear flows appearing as the larger structure (as zonal flows) the process is most effective [26, 29-32]. Recently, this process has been directly observed in configuration space with fast imaging [33]. As the nonlocal inverse kinetic energy transfer takes place as the dominant mechanism (Fig. 1) for interaction among eddies and does not include noncoherent fluctuations, this explanation is further supported.

Interactions between random fluctuations are shown in Fig. 4. The transfer is about an order of magnitude lower and furthermore does not show a preferred direction. The interaction among noncoherent fluctuations is therefore random and does not yield to coherence in the nonlinear coupling and subsequently to no significant intermodal energy transfer. This supports the work by Bruneau [17] where it is shown that in neutral $2 \mathrm{D}$ fluids eddies are responsible for the inverse energy trans-

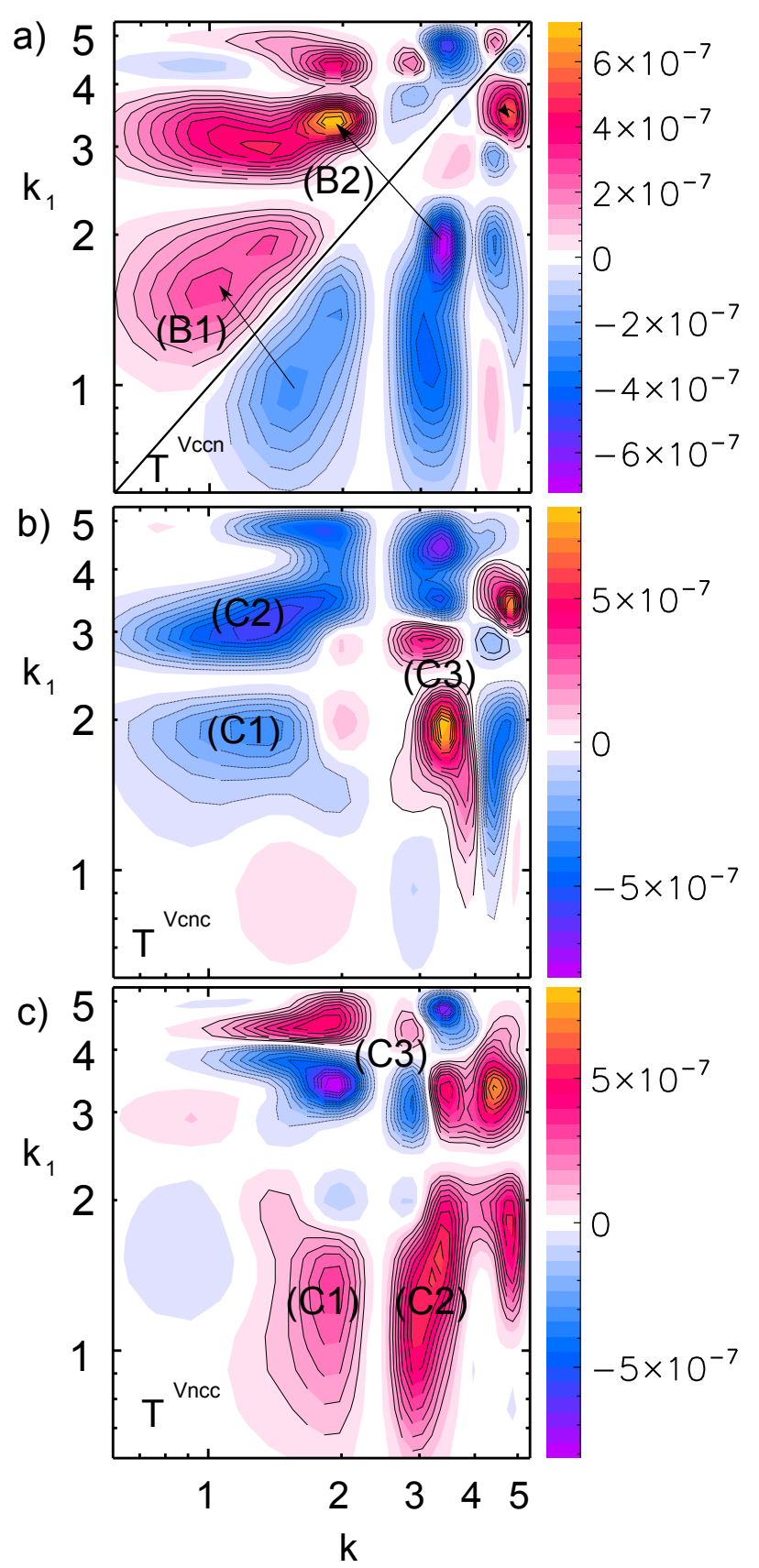

FIG. 5: Kinetic energy transfer between two coherent and one noncoherent structure. Within this process the energy is transferred between the two coherent structures (a), from the noncoherent into the coherent (b) and from the coherent into the noncoherent fluctuations (c).

fer. However, the conclusion that noncoherent structures do not significantly participate in the nonlinear coupling process is misleading as we will see in the following two sections. 

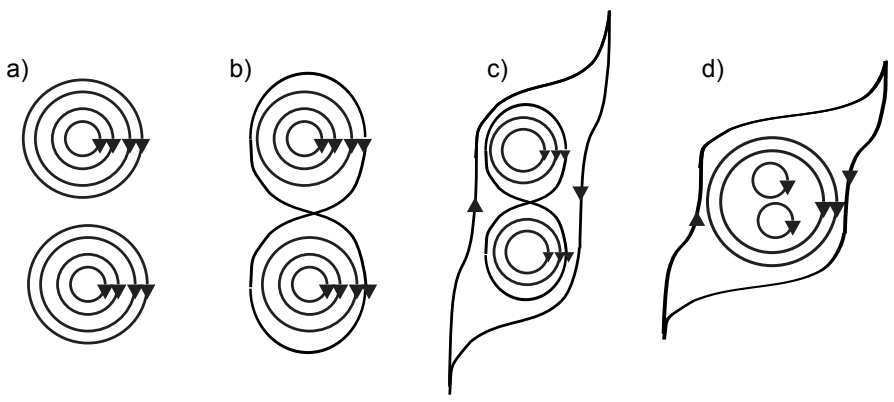

FIG. 6: Artist view of the vortex merger process.

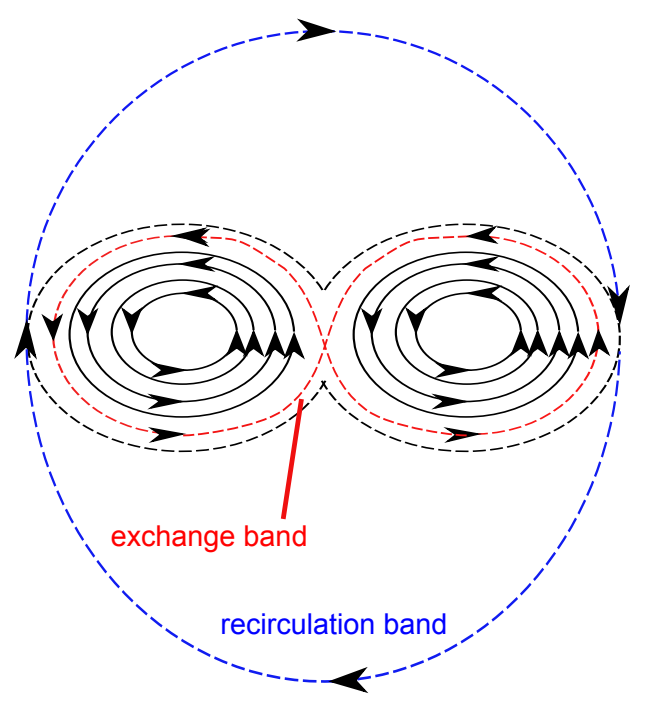

FIG. 7: Schematic streamline configuration of a vortex merger.

\section{VORTEX MERGER}

To investigate the interaction of the coherent and noncoherent field two cases can be distinguished. In the first two coherent modes couple with a noncoherent mode. In the second two noncoherent modes couple with one coherent mode. The first case is shown in the Fig. 5. The energy transfer between different coherent scales $T^{V c c n}\left(\mathbf{k} \leftarrow \mathbf{k}_{\mathbf{1}}\right)$ in the presence of noncoherent scales is inverse and local as shown by the arrows (B1) and (B2) in Fig. 5a. The energy is conservative as seen by the asymmetry around the $k=k_{1}$ line due to $T^{V c c n}\left(\mathbf{k} \leftarrow \mathbf{k}_{\mathbf{1}}\right)=-T^{V c c n}\left(\mathbf{k}_{\mathbf{1}} \leftarrow \mathbf{k}\right)$. The common vortex merger is representative of a local event by merging two (corotating) eddies of the same size to an eddy of nearly twice the size. However, one would assume this process is taking place within interaction among only coherent structures (Fig. 2). To understand why this process is observed here as a cross-field transfer a closer look on the anatomy of the flow field of vortex mergers [19] can reveal some insight.

The vortex merging process consists of three phases

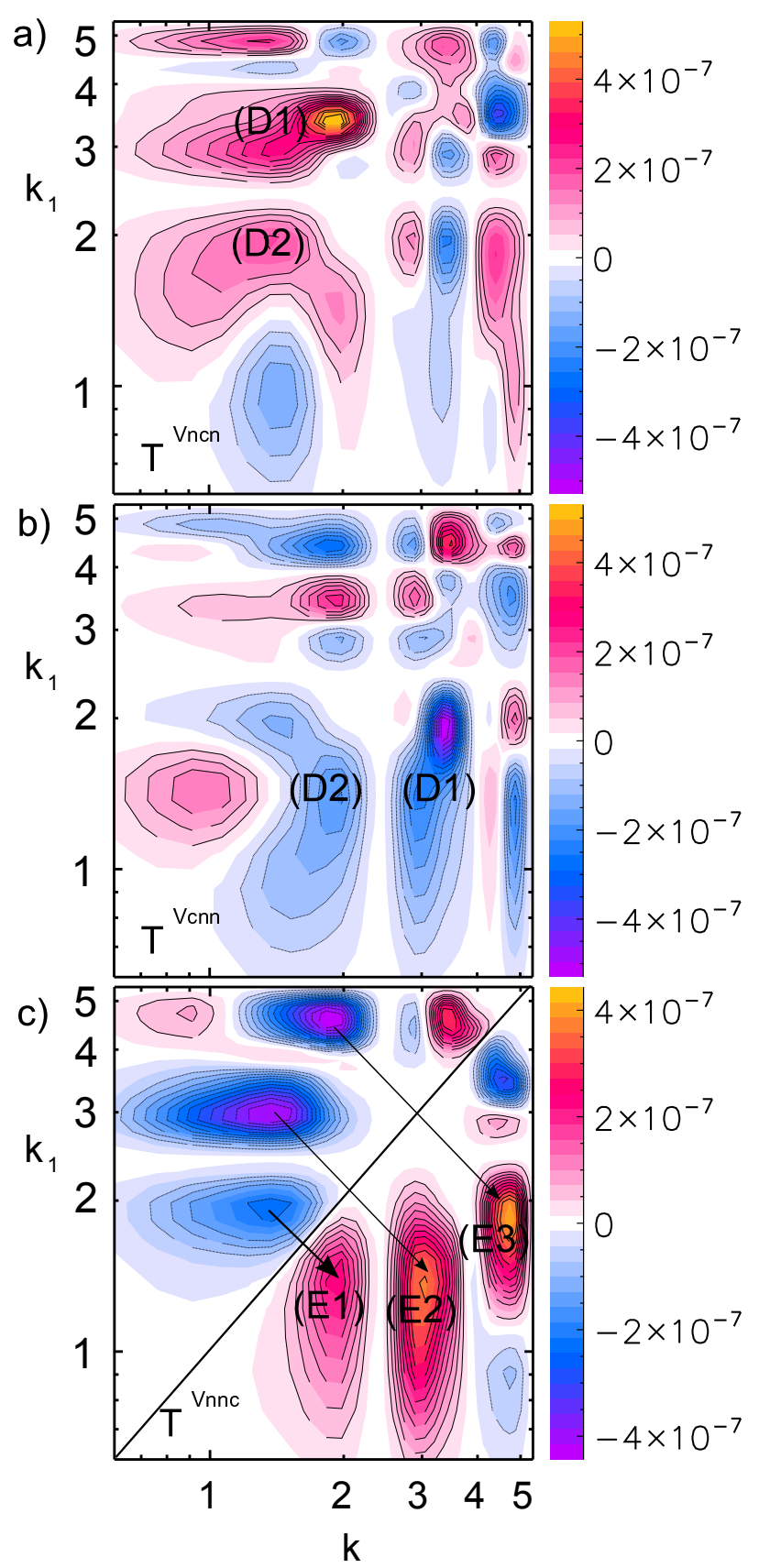

FIG. 8: Kinetic energy transfer between one coherent and two noncoherent structures. Within this process the energy is transferred from the coherent into the noncoherent (b) and from the noncoherent into the coherent fluctuations (c) and between the two noncoherent structures (c).

$[18,19]$. In the first phase the vortex cores grow by viscous diffusion where the distance between the vortices stays constant (Fig. 6a and b). If the vortex cores reach a critical size the distance decreases by convection and a formation of filaments occurs (Fig. $6 \mathrm{~b}$ and c). This is the heart of the vortex merging process and these filaments are noncoherent structures. In the last phase 
a)

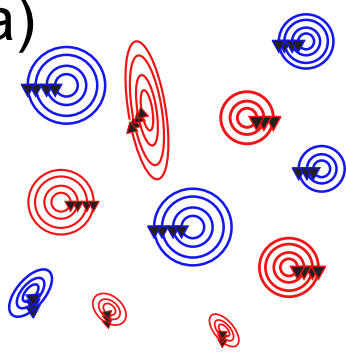

FIG. 9: Schematics of the vortex clustering process. A random spatial distribution of vortices (a) self-organize, where concentrations of same-sign vortices appear as larger noncoherent structures (b).

the vorticity maxima are reduced by diffusion (Fig. 6d). Furthermore, the appearance of the noncoherent structure in the vortex merging process can be understood by considering the streamline configuration of the vortex merger $[18,19]$ as depicted in Fig. 7 . The flow consists of two inner core regions (these are the two eddies), the exchange band and two outer recirculation regions. Vorticity which enters the recirculation bands leads to the formation of these filaments. Vorticity from one eddy can enter the exchange band. The exchange band constitutes a filamentary structure. The vorticity within the exchange-band can be absorbed into the other eddy. In any case the vortex merger process is an interaction of a coherent structure (one of the inner cores) with a noncoherent structure (the exchange band or the recirculation region). Vortex merging has to transfer energy in the noncoherent field and is therefore directly linked to the transfer of noncoherent energy $T^{V n c c}\left(\mathbf{k} \leftarrow \mathbf{k}_{\mathbf{1}}\right)$.

But before we have a look on this, let us consider the transfer from or into the coherent field $T^{V c n c}\left(\mathbf{k} \leftarrow \mathbf{k}_{\mathbf{1}}\right)$ first. As seen in Fig. 5b energy in the coherent field at large scales is lost to noncoherent structures at small scales $((\mathrm{C} 1)$ and $(\mathrm{C} 2))$, which could be the filamentary structure in the exchange band or the filaments in the outer recirculation region. Furthermore, coherent structures gain energy from noncoherent structures at small but similar scales (the red region (C3) in $T^{V c n c}$ ). This process displays the alignment of random vortices giving them a preferred direction which in turn increases the coherent vortices at larger scale. However, this mechanism is not as important as the vortex merger. The energy transfer in Fig. $5 \mathrm{~b}$ is not asymmetric around the line $k=k_{1}$, however, the process is still conservative. Because it is a cross-field transfer $T^{V c n c}$ has to be studied together with $T^{V n c c}$. Considering $T^{V n c c}\left(\mathbf{k} \leftarrow \mathbf{k}_{\mathbf{1}}\right)$ in Fig. $5 \mathrm{c}$ the balance comes out likewise. Due to the interactions of two coherent and one noncoherent structure the noncoherent field is strongly driven by coherent fluctuations at large scales. Because all these observations are related to the same three-wave coupling process the local inverse energy cascade of coherent structures is directly bound to a transfer of large-scale coher- ent energy to small-scale noncoherent energy. Therefore coherent energy is transferred to larger coherent scales and to small noncoherent structures. Even though large coherent structures are formed the overall process just transfers energy from small-scale coherent into smallscale noncoherent structures using the coherent field as an interim energy reservoir. The transfer of energy into the noncoherent field can be seen as dissipation and an increase in entropy. Therefore vortex merging not only decreases it also partially increases the entropy.

\section{VORTEX CLUSTER}

Just by the superposition of the vorticity field (as shown in Fig. 9a) cluster of same-sign vorticies constitutes a large-scale strain field, which can interact with other structures. A cluster does not exhibit closed streamlines and is therefore a large-scale noncoherent structure consisting of small-scale coherent structures (eddies). The self-organization into a cluster should tranfer energy from the small-scale coherent into the largescale noncoherent field. The coupling between two noncoherent and one coherent mode is shown in Fig. 8. As seen in Fig. 8a coherent fluctuations at smaller scales transfer energy into the large-scale noncoherent field (D1). This large-scale noncoherent structure is not an eddy but it constitutes a strain field. The constant energy supply by eddies at multiple smaller scales let suggest that this large-scale strain field is indeed a cluster. As the process investigated in the last section also the energetic interactions between the noncoherent and coherent field are directly coupling to other interactions. Therefore as energy is transferred from small-scale coherent to largescale noncoherent fluctuations (Fig. 8a) there must be a loss of coherent energy at small scales to noncoherent fluctuations as seen in Fig. 8b. Also here a cascade within the noncoherent energy is involved (Fig. 8c). Here kinetic energy is transferred from large to small scales. Left to themselves without energy supply from the coherent field the vortex cluster cannot suistain itself. This is a result of the rather weak self-rotation, remember the vorticity is smaller than the strain as $\mathcal{Q}>0$, and therefore the eddies within the vortex cluster will diffuse out of the cluster and the cluster is splitting up in smaller and smaller ones. In summary, coherent energy is transferred to larger noncoherent scales and to small noncoherent structures. Even though large noncoherent structures are formed the overall process transfers energy from small-scale coherent into small-scale noncoherent structures as in the case of the vortex merger. Therefore vortex clustering increases the entropy.

\section{CONCLUSION}

The linear drive in two-dimensional turbulence provides a constant source of coherent kinetic energy into 
the system. Since the kinetic energy in two-dimensional turbulence cannot be dissipated as in three dimensions, the turbulence has to find a way to transfer the coherent energy into the noncoherent field. The cross-field kinetic energy transfer between the different scales of coherent and noncoherent motion in two-dimensional drift-wave turbulence in a confined magnetized plasma has been investigated. It has been shown that large-scale turbulent structures are generated by vortex merging, thinning and clustering. The process of vortex merging constitutes an inverse cascade process of coherent energy, which is accompanied by a transfer from large-scale coherent to small-scale noncoherent energy (negative coherence production at large scales). Large-scale strain fields can be generated by an alignment of coherent structures, where energy is transferred from small-scale coherent to largescale noncoherent fluctuations. This negative coherence production at small scales is coupled to a direct cascade of noncoherent energy resulting in dissipation. It is therefore convenient for two-dimensional turbulent systems or it is even required that large-scale turbulent structures are generated due to vortex merging and clustering to transfer energy from the coherent into the noncoherent field as a way to dissipate coherent energy. Nonlocal energy transfer by vortex thinning appear to be the strongest process in structure formation in drift-wave turbulence.
[1] G. Boffetta and R. E. Ecke, Annu. Rev. Fluid. Mech. 44, 427 (2011).

[2] J. Paret and P. Tabeling, Phys. Fluids 10, 3126 (1998).

[3] S. Chen, R. E. Ecke, G. L. Eyink, M. Rivera, M. Wang, and Z. Xiao, Phys. Rev. Lett. 96, 084502 (2006).

[4] J.-Z. Wu, H.-Y. Ma, and M.-D. Zhou, Vorticity and Vortex Dynamics (Springer, Berlin Heidelberg, 2006).

[5] P. Manz, M. Ramisch, and U. Stroth, Plasma Phys. \& Controlled Fusion 51, 035008 (2009).

[6] U. Stroth, F. Greiner, C. Lechte, N. Mahdizadeh, K. Rahbarnia, and M. Ramisch, Phys. Plasmas 11, 2558 (2004).

[7] S. J. Camargo, D. Biskamp, and B. D. Scott, Phys. Plasmas 2, 48 (1995).

[8] P. Manz, M. Ramisch, U. Stroth, V. Naulin, and B. D. Scott, Plasma Phys. \& Controlled Fusion 50, 035008 (2008).

[9] S. Enge, G. Birkenmeier, P. Manz, M. Ramisch, and U. Stroth, Phys. Rev. Lett. 105, 175004 (2010).

[10] A. Köhn, G. Birkenmeier, E. Holzhauer, M. Ramisch, and U. Stroth, Plasma Phys. \& Controlled Fusion 52, 035003 (2010).

[11] M. Ramisch, N. Mahdizadeh, U. Stroth, F. Greiner, C. Lechte, and K. Rahbarnia, Phys. Plasmas 12, 032504 (2005).

[12] N. Mahdizadeh, F. Greiner, M. Ramisch, U. Stroth, W. Guttenfelder, C. Lechte, and K. Rahbarnia, Plasma Phys. \& Controlled Fusion 47, 569 (2005).

[13] N. Mahdizadeh, F. Greiner, T. Happel, A. Kendl, M. Ramisch, B. D. Scott, and U. Stroth, Plasma Phys. \& Controlled Fusion 49, 1005 (2007).

[14] A. Okubo, Deep-Sea Res. 17, 445 (1970).

[15] D. Weiss, Physica D 48, 273 (1991).

[16] V. Naulin and K. H. Spatschek, Phys. Rev. E 55, 5883 (1997).

[17] C. H. Bruneau, P. Fischer, and H. Kellay, EPL 78, 34002
(2007).

[18] C. Cerretelli and C. H. K. Williamson, J. Fluid. Mech. 475, 41 (2003).

[19] L. K. Brandt and K. K. Nomura, Phys. Fluids 18, 051701 (2006).

[20] D. A. D'Ippolito, J. R. Myra, and S. J. Zweben, Physics of Plasmas 18, 060501 (2011)

[21] A. Hasegawa and M. Wakatani, Phys. Rev. Lett. 50, 682 (1983).

[22] A. Hasegawa, C. G. Maclennan, and Y. Kodama, Phys. Fluids 22, 11 (1979).

[23] F. Y. Gang, B. D. Scott, and P. H. Diamond, Phys. Fluids B: Plasma Phys. 1, 1331 (1989).

[24] A. J. Domaradzki, Phys. Fluids 31, 2747 (1988).

[25] M. Wakatani and A. Hasegawa, Phys. Fluids 27, 611 (1984).

[26] P. Manz, M. Ramisch, and U. Stroth, Phys. Rev. Lett. 103, 165004 (2009).

[27] S. Kida, J. Phys. Soc. Jpn. 50, 3517 (1981).

[28] G. L. Eyink, Physica D 207, 91 (2005).

[29] M. Ramisch, G. Birkenmeier, T. Happel, A. Köhn, N. Mahdizadeh, P. Manz, B. Nold, R. Wilcox, D. T. Anderson, and U. Stroth, Contr. Plasma Phys. 50, 718 (2010).

[30] P. Manz, M. Ramisch, and U. Stroth, Phys. Rev. E 82, 056403 (2010).

[31] M. Ramisch, P. Manz, U. Stroth, G. Birkenmeier, S. Enge, E. Holzhauer, A. Köhn, and B. Nold, Plasma Phys. \& Controlled Fusion 52, 124015 (2010).

[32] U. Stroth, P. Manz, and M. Ramisch, Plasma Phys. \& Controlled Fusion 53, 024006 (2011).

[33] M. Xu, G. R. Tynan, P. H. Diamond, S. H. Müller, C. Holland, J. H. Yu, and Z. Yan, Phys. Rev. Lett. 107, 055003 (2011). 